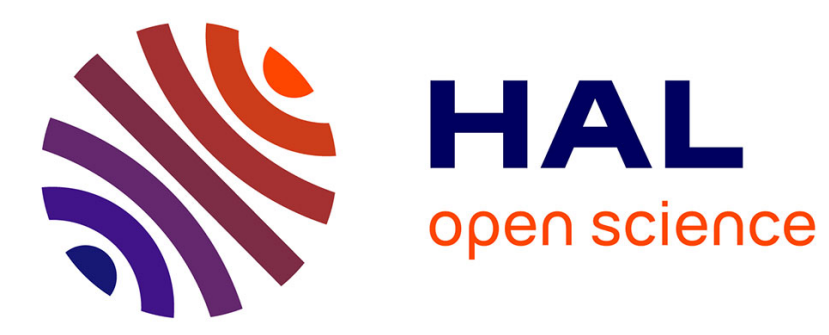

\title{
Lateral resolution of electrostatic force microscopy for mapping of dielectric interfaces in ambient conditions
}

M Labardi, A Bertolla, Cyrille Sollogoub, R Casalini, S Capaccioli

\section{To cite this version:}

M Labardi, A Bertolla, Cyrille Sollogoub, R Casalini, S Capaccioli. Lateral resolution of electrostatic force microscopy for mapping of dielectric interfaces in ambient conditions. Nanotechnology, 2020, 31 (33), pp.1-9. 10.1088/1361-6528/ab8ede . hal-02902738

\section{HAL Id: hal-02902738 \\ https://hal.science/hal-02902738}

Submitted on $20 \mathrm{Jul} 2020$

HAL is a multi-disciplinary open access archive for the deposit and dissemination of scientific research documents, whether they are published or not. The documents may come from teaching and research institutions in France or abroad, or from public or private research centers.
L'archive ouverte pluridisciplinaire HAL, est destinée au dépôt et à la diffusion de documents scientifiques de niveau recherche, publiés ou non, émanant des établissements d'enseignement et de recherche français ou étrangers, des laboratoires publics ou privés. 


\title{
Lateral resolution of electrostatic force microscopy for mapping of dielectric interfaces in ambient conditions
}

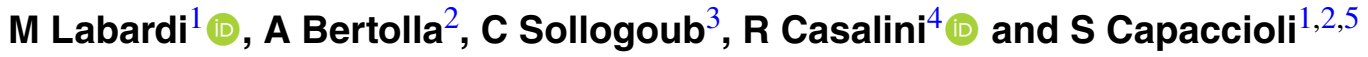 \\ ${ }^{1}$ CNR-IPCF, Sede Secondaria di Pisa, Largo Pontecorvo 3, 56127, Pisa, Italy \\ ${ }^{2}$ Dipartimento di Fisica 'E. Fermi', Università di Pisa, Largo Pontecorvo 3, 56127, Pisa, Italy \\ ${ }^{3}$ PIMM, Arts et Metiers Institute of Technology, CNRS, Cnam, HESAM University, 151 Bd. de \\ l'Hopital, 75013, Paris, France \\ ${ }^{4}$ Chemistry Division, Naval Research Laboratory, Washington, DC 20375-5320, United States of \\ America \\ ${ }^{5}$ CISUP, Centro per l'Integrazione della Strumentazione dell'Università di Pisa, Lungarno Pacinotti 43, \\ 56126, Pisa, Italy
}

E-mail: labardi@df.unipi.it

\begin{abstract}
The attainable lateral resolution of electrostatic force microscopy (EFM) in an ambient air environment on dielectric materials was characterized on a reference sample comprised of two distinct, immiscible glassy polymers cut in a cross-section by ultramicrotomy. Such a sample can be modeled as two semi-infinite dielectrics with a sharp interface, presenting a quasi-ideal, sharp dielectric contrast. Electric polarizability line profiles across the interface were obtained, in both lift-mode and feedback-regulated dynamic mode EFM, as a function of probe/surface separation, for different cases of oscillation amplitudes. We find that the results do not match predictions for dielectric samples, but comply well or are even better than predicted for conductive interfaces. A resolution down to $3 \mathrm{~nm}$ can be obtained by operating in feedback-regulated EFM realized by adopting constant-excitation frequency-modulation mode. This suggests resolution is ruled by the closest approach distance rather than by average separation, even with probe oscillation amplitudes as high as $10 \mathrm{~nm}$. For better comparison with theoretical predictions, effective probe radii and cone aperture angles were derived from approach curves, by also taking into account the finite oscillation amplitude of the probe, by exploiting a data reduction procedure previously devised for the derivation of interatomic potentials.
\end{abstract}

Keywords: electrostatic force microscopy, lateral resolution, dielectrics, interface

(Some figures may appear in colour only in the online journal)

\section{Introduction}

Electrostatic force microscopy (EFM) is a generic term for scanning probe methods based on atomic force microscopy (AFM), where the conductive sharp probe is electrically biased, and the effect of electrostatic forces between the tip and sample is detected. EFM can be exploited to obtain information on various electrical properties of a surface, such as dielectric polarizability, surface bound charge, and contact potential, on a local scale. Some relevant applications concern the mapping of nanoinclusions in dielectric matrices of nanocomposites [1,2], detection of injected charges on conductive nanolayers [3], and characterization of semiconductor devices and junctions [4]. Of particular interest is the EFM application to realize local dielectric spectroscopy [5], realized with different variants [6-8]. This could potentially allow discrimination 


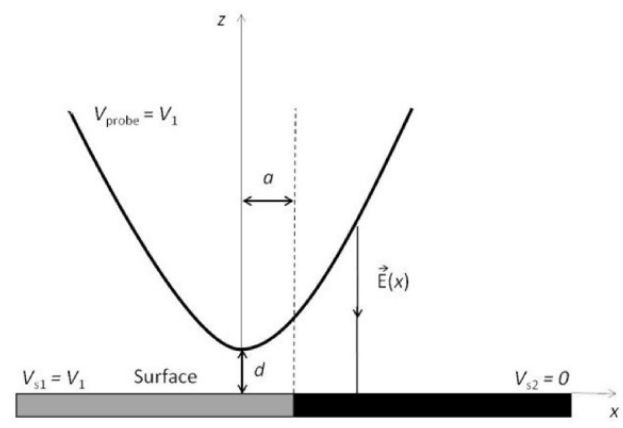

(a)

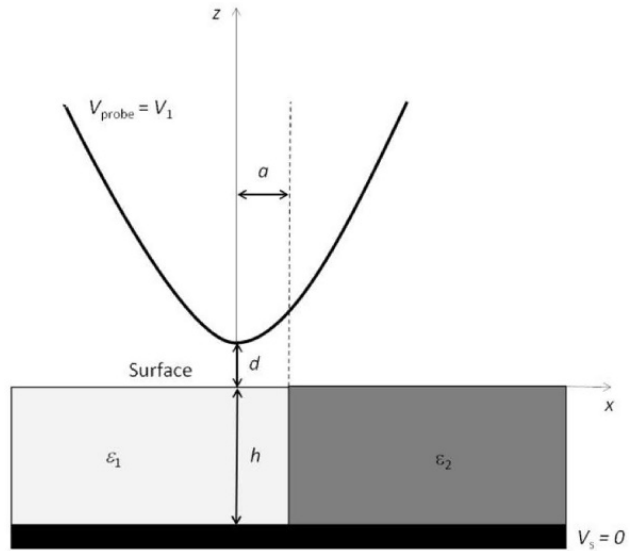

(b)

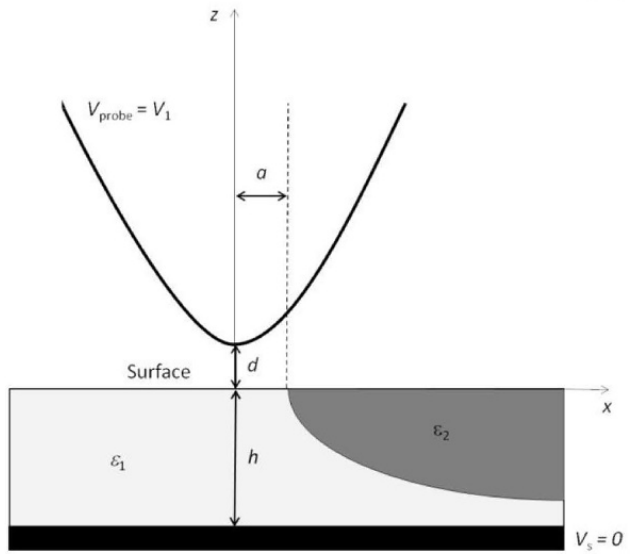

(c)

Figure 1. (a) Sketch of probe/surface geometry for calculation of resolution (as from [4]). (b) Case of two dielectric materials with ideal geometry (symmetry along $z$ ), of dielectric constants $\varepsilon_{1}$ and $\varepsilon_{2}$, and thickness $h$. (c) Case of two dielectric materials with unknown buried geometry.

of dielectric relaxation phenomena on the characteristic length scale of dynamical heterogeneity [9], which in glass-forming materials such as polymers amounts to a few nm [10,11].

The long-range nature of Coulombic forces limits attainable spatial resolution to several nanometers, in opposition to the much higher resolution allowed by short-range forces in AFM, due to chemical interactions and atomic mutual repulsion, which pushes the limit down to a sub-atomic scale [12]. Reports of EFM resolution down to the atomic scale are available, in ultra-high vacuum experiments and concerning surface atomic dipole interactions [13]. Resolution on step-like

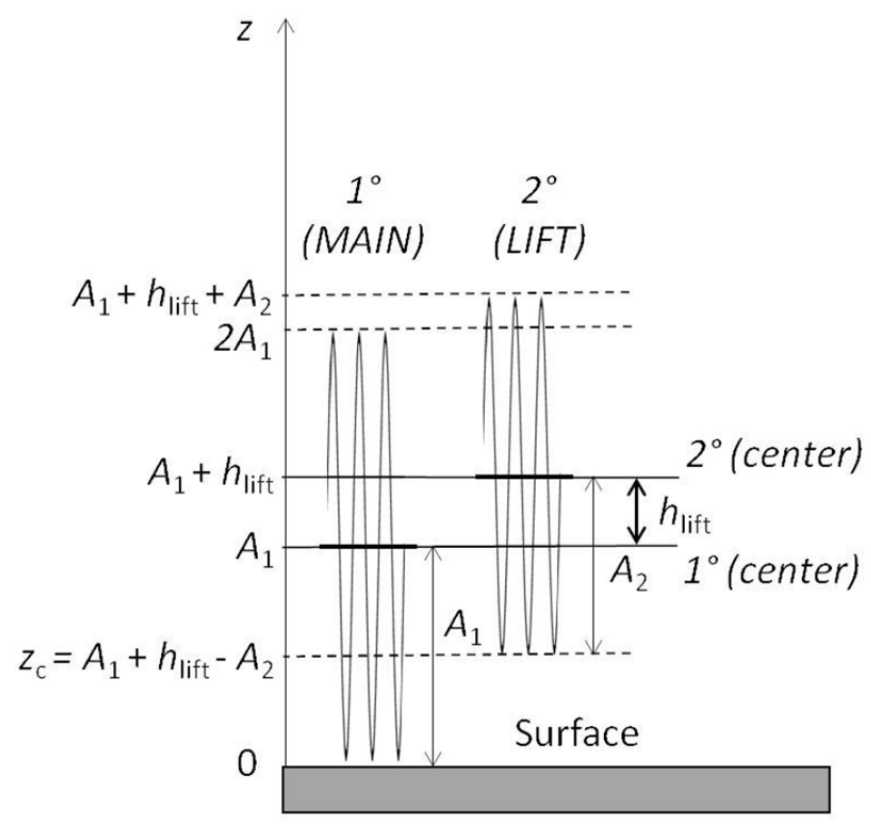

Figure 2. Sketch of the probe oscillation amplitude during feedback-regulated operation (main, amplitude $A_{1}$ ) and lift mode (lift height $h_{\text {lift }}$, amplitude $A_{2}$ ).

boundaries between insulating salt layers and the metal substrate beneath has also been assessed by comparing EFM detection modes based on force and force gradient [14]. Such a refined performance is more difficult to achieve in ambient environments, where surfaces are typically contaminated by adsorbed moisture layers. Nevertheless, physical quantities, e.g. dielectric constant, contact potential difference, and surface charge density, can be relevant for characterizing a surface on a nanometer length scale, where a description in terms of band theory and thermodynamics can still be appropriate. The efforts to improve the resolution of local electrical probe techniques in these systems is due to the necessity of characterizing functional properties of increasingly small electronic devices and junctions, as well as inspecting diffuse interfaces in nanocomposite materials, for energy or sensing applications.

The EFM spatial resolution $\delta$ can be described, according to well-accepted models with a probe with cylindrical symmetry and a parabolic profile [15], in terms of an apex probe radius $R$ and tip/surface distance $d$, as, for instance:

$$
\delta=\frac{8}{3} \sqrt{2 R d}
$$

This is valid in the case of a small distance, $d \ll R$, and by assuming, as a resolution criterion, that $\delta$ is the lateral displacement necessary to obtain a change of the signal of interest from $10 \%$ to $90 \%$ of the total change across a discontinuity.

Spatial resolution is typically determined by calculating the electrostatic force between a conductive probe with cylindrical symmetry and a heterogeneous flat sample, divided into two parts which have distinct electric properties, ideally by a sharp interface, as sketched in figure 1(a). The calculation is usually simplified by resorting to the Derjaguin approximation 
Table 1. Nominal specifications of the probes used. $k$ is the nominal cantilever spring constant, $f_{0}$ the measured free resonant frequency.

\begin{tabular}{llllrr}
\hline Label & Brand & Model & Material & $k(\mathrm{~N} / \mathrm{m})$ & $f_{0}(\mathrm{kHz})$ \\
\hline$‘ \chi$ & NanoSensors & PPP-NCLPt & PtIr5 & 47 & 158.03 \\
$' \eta$ ' & MikroMasch & HQ:NSC16/Cr-Au & Au & 45 & 139.24 \\
\hline & MikroMasch & HQ:DPER-XSC11 & Pt & 42 & 355.32 \\
\hline
\end{tabular}

[16], that is, assuming all electric field lines are parallel to the direction of symmetry. By calculating the electrostatic force as a function of the lateral position of the probe with respect to the location of the interface, a force profile is obtained, whose width provides the resolution $\delta$, as a function of distance $d$ and probe radius $R$. On the condition that $d \ll R$, the Derjaguin approximation provides a good description of the problem [15].

Another common definition for the EFM resolution is given in terms of the capability to resolve two point-like objects on the surface [17-19], or in other words, in terms of the width of a point spread function. However, we prefer to adhere to the former definition, for the unidimensional case, which allows comparisons with previous results, e.g. the ones reported in $[14,15]$.

Contrary to what is obtained for metals, with dielectric materials the electric field penetrates below the surface, and polarization can be induced at buried interfaces, such as the ones that can be present in composite materials [20]. Therefore, the bulk structure of a sample can also contribute to influencing imaging resolution, unlike in the case of conducting interfaces. This feature is commonly exploited, for instance, for imaging of sub-surface nanoinclusions in polymeric nanocomposites $[1,2]$. Furthermore, the thickness of the dielectric layer $h$ may be large compared to $d$, as sketched in figure 1(b), therefore the Derjaguin approximation becomes insufficient for a correct theoretical description. Indeed, estimations in this case are usually carried out by numerical simulation.

With regard to lateral resolution, defined as the passage through the step boundary in a dielectric sample structure with translational symmetry along the $z$-axis, akin to the one in figure 1(b), numerical simulations could be, in principle, performed. However, in the case of more realistic samples, the three-dimensional geometry of the interface could be different, e.g. like that depicted in figure 1(c), where phase separation occurs with an unknown geometry, buried below the surface. Imaging of this situation by EFM may lead to deterioration of resolution, due to the thickness change of the component materials within the probed volume. For a reliable assessment of the limit imaging resolution, the latter effect should be avoided, therefore a sample with the structure of figure 1(b) should be used, for the most appropriate comparison with estimations.

Another critical point to consider for the most correct estimation of EFM resolution is related to the probe shape. It is well known that in EFM, interaction of other probe parts (such as the probe shaft and the AFM cantilever) leads to resolution loss [21]. A force gradient detection method is usually adopted to contrast such degradation, yielding remarkable rejection of stray contributions, while on the other hand, the bare force detection is strongly affected by the influence of the extending parts of the probe, like the tip shaft and the cantilever [21,22].

The standard scanning probe operation mode for the detection of force gradients is the frequency-modulated (FM)-AFM [23], where the shift $\Delta f$ of the resonant frequency of the cantilever oscillation provides information on the force gradient magnitude. Yet, some contributions from the closest part of the probe shaft also persist by using such a mode. For this reason, in order to obtain the most reliable information on the sample from EFM measurements, the probe geometry should be known to the highest level of detail.

The usual method to characterize the shape of conductive probes is imaging by scanning electron microscopy (SEM). Inspection of common EFM probes reveals that their geometry is not perfectly reproducible from probe to probe; in particular, the curvature of the shaft close to the apex, as well as the symmetry at the probe edge, are usually not uniform (as visible for instance from the SEM pictures shown in figure 3). Although SEM characterization is important, particularly for checking the probe integrity, or the presence of debris after operation, calibration procedures of the probe performance, concerning specifically electric force detection, are usually carried out by means of the EFM itself. They consist of recording approach curves, where the frequency shift is measured as a function of the probe/surface separation $L$, by using a flat metallic sample [24], and then by best fitting to functions obtained by analytical or numerical calculations, assuming a given probe shape parametrization. This procedure provides effective shape parameters for the probe, and releases the necessity of imaging the AFM tips ex-situ.

The probe shape can be described in different ways, the most common ones being either a paraboloid shape with apex radius $R[15,21]$, or a spherical cap with apex radius $R$ and a truncated cone with shaft angle $\theta_{0}[6,25,26]$. In this work, the last probe shape will be assumed, being more commonly used.

\section{Materials and methods}

In order to investigate the inherent limit resolution of EFM on dielectric materials in ambient conditions, we have devised a quasi-ideal dielectric step-contrast surface, where the interface extends in the normal direction through the whole sample thickness. To realize it, we have used a polymer multi-layered film of polystyrene (PS) and poly(lactic acid) (PLA) [27], two immiscible polymers in glassy state at room temperature, but with different polar characters. The multilayer period was chosen to be rather large (around $25 \mu \mathrm{m}$ ), in order to obtain well separated interfaces. The multilayer was cross-sectioned by ultramicrotomy, after embedding in microtome resin, by 


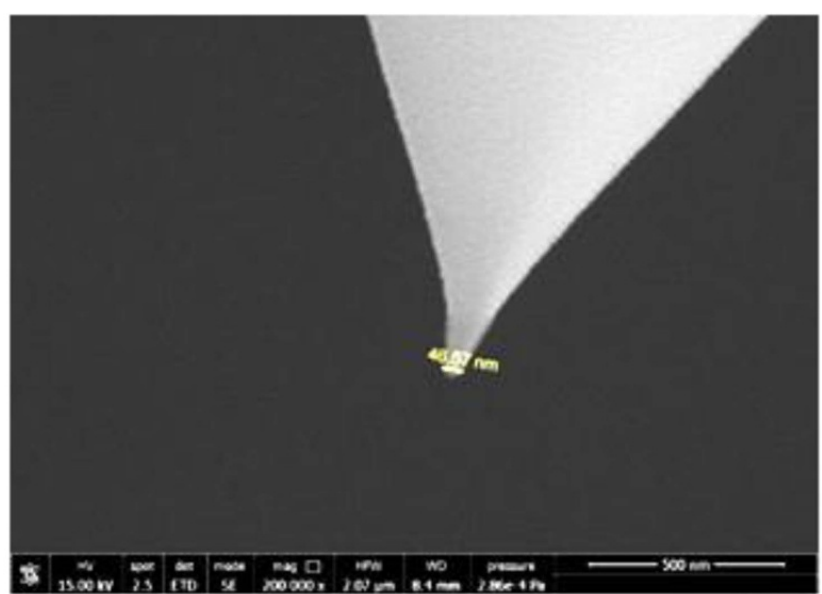

(a)

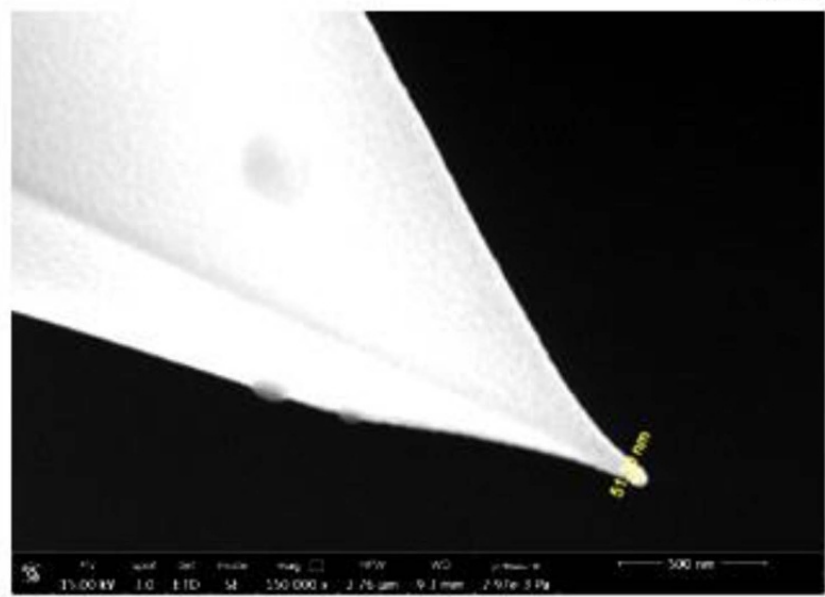

(b)

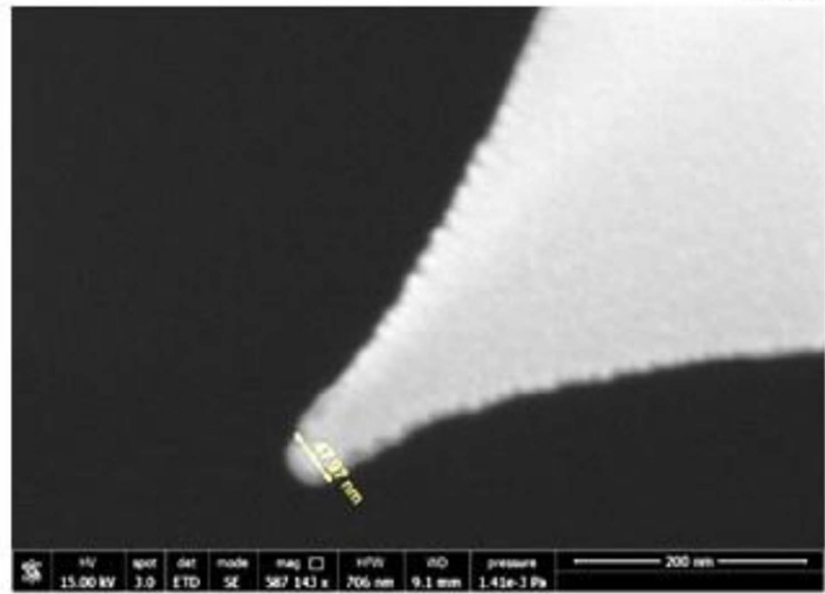

(c)

Figure 3. SEM micrographs of the three probes used: (a) probe ' $\chi$ ', (b) probe ' $\eta$ ', (c) probe ' $\xi$ ' (the dimension determined by the SEM micrographs are reported in table 2). Images were taken after use.

choosing the cutting direction at $45^{\circ}$ with respect to the interface direction, for easier discrimination of those topographic contributions due to the cut from the ones due to the interface itself. The obtained section was applied on a metal sample holder, with the result about 1-2 mm thick.

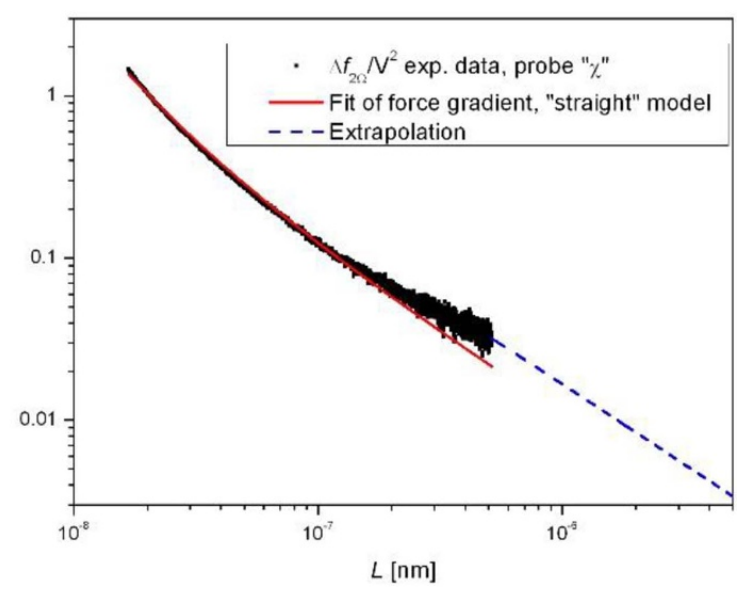

Figure 4. Approach curve for probe ' $\chi$ ', with best fit (red solid line) by the 'straight' model (equation (2)) for the force gradient [26]. The extrapolated function to allow data integration according to equation (3) is also shown (blue dashed line).

For both the force/distance curves and interface profiling, a Veeco Instruments NanoScope IIIa AFM with a MultiMode head was used. EFM was performed by adopting an external AFM controller for frequency modulation (PLLProII, RHK Technology, Troy, USA), based on a phase-locked loop (PLL) detector, optimized to improve EFM performance for local dielectric spectroscopy applications [7, 8]. A voltage $V(t)=V_{\mathrm{ac}} \cos (\Omega t)$ was applied to the conductive probes, at a frequency $\Omega(\sim 80 \mathrm{~Hz})$, and $V_{\mathrm{ac}}=15 \mathrm{~V}_{\mathrm{RMS}}$. This rather high value of $V_{\text {ac }}$ is necessary for operation on thick dielectric samples compared to thin films, in order to obtain a high enough response of the material. Nonetheless, the resulting electric field remains low enough to avoid the onset of nonlinear effects in dielectrics. The value of $\Omega$ is chosen as a compromise between the scanning speed and $\mathrm{S} / \mathrm{N}$ ratio, as in the previous literature $[2,7,28]$.

The frequency shift output $\Delta f(t)$ of the PLL controller was demodulated, at frequency $2 \Omega$, by a dual lock-in amplifier (SRS830DSP, Stanford Research Systems, USA). The amplitude $\Delta f_{2} \Omega$ of such frequency shift modulation depends only on the probe/sample capacitance at the current probe position, with no influence from both static charges and contact potential difference [7], allowing more accurate estimations of the dielectric permittivity. All measurements were performed at room temperature and in dry nitrogen gas atmosphere conditions.

Two different scanning modes were used, namely the liftmode, customarily used in EFM spectroscopic applications [6], and the FM-AFM at constant excitation [29], using the oscillation amplitude as the signal for distance regulation feedback [30], by means of the same PLL controller. We choose to adopt the latter scheme with the aim of improving the lateral resolution of EFM measurements in ambient conditions. Indeed, the use of frequency shift feedback regulation, employed in alternative schemes [31], is usually more critical to operate in such conditions.

In both AFM operation modes, electric force is detected by recording the frequency shift of the cantilever oscillation 
Table 2. Results of best fit with the 'straight' model for the three probes, in the cases of gradient model [26] and force model by inversion (introduced here). Values of shaft angle transpire as more consistent with the geometry resulting from SEM imaging for the latter model. Note that $\theta_{0}$ had an upper constraint of 1 rad in these fittings; this was however only reached in the case of the gradient model.

\begin{tabular}{|c|c|c|c|c|c|c|}
\hline Label & $R_{\mathrm{SEM}}(\mathrm{nm})$ & $\theta_{0, \mathrm{SEM}}$ (rad) (approx.) & $R_{\text {FIT }}(\mathrm{nm})$ (gradient) & $\theta_{0, \text { FIT }}($ rad $)($ gradient $)$ & $R_{\mathrm{FIT}}(\mathrm{nm})$ (force) & $\theta_{0, \mathrm{FIT}}(\mathrm{rad})$ (force) \\
\hline$' x '$ & 23.3 & $0.3-0.45$ & 23.2 & 0.97 & 33.4 & 0.48 \\
\hline ' $\eta '$ & 25.8 & 0.35 & 57.8 & 1 (limit) & 42.9 & 0.57 \\
\hline ' $\xi$ ' & 23.9 & $0.35-0.45$ & 20.3 & 1 (limit) & 22.8 & 0.49 \\
\hline
\end{tabular}

caused by the electrostatic force field. In the feedbackregulated mode, an oscillation amplitude $A_{1}$ is held constant by the distance feedback regulation (see sketch in figure 2), while the tip is concerned also by atomic forces at its oscillation edge, the main ones in ambient conditions being a result of the capillarity of adsorbed layers, and to atomic repulsion. Such an interaction will lead to a shift $\Delta f_{\text {at }}$ as a result of atomic interactions, which is also measured by the PLL controller. If an electric potential is applied, an additional frequency shift $\Delta f_{\text {el }}$ will occur according to the applied bias. In the lift mode, a topographic profile is recorded during a first (main) pass, in the same way, while applying no bias; during the second (lift) pass, the same profiling is then reproduced at a lifted height, with no need for feedback control. Therefore, during the second pass, the tip is no longer subjected to the atomic forces, but only to the electric field produced by the probe biasing. The tip oscillation $A_{2}$ during the lift can be changed arbitrarily, from very small to as wide as the tip/sample separation $L=A_{1}+h_{\text {lift }}$ (figure 2); if this condition is exceeded, the tip could impact the surface in an uncontrolled way, so this is not usually done. The closest approach distance $z_{\mathrm{c}}$ is nearly zero under distance feedback-regulation, while it amounts to $A_{1}-A_{2}+h_{\text {lift }}$ during the lift mode second pass (figure 2).

\section{Results and discussion}

\subsection{Probe calibration}

We have compared results from three widespread models of AFM tips with a pyramidal shaft, listed in table 1. SEM pictures of the probes are shown in figure 3. Approach curves to a flat gold surface, deposited on glass, were recorded for each of these probes, in order to determine their effective probe radius and shaft angle. An example of such an approach curve for probe type ' $\chi$ ' is shown in figure 4.

Best fitting by one of the models described in [26], the socalled 'straight' model, which emerged here as better modeling our data, was used:

$$
\begin{aligned}
F_{S}^{a}= & -\pi \varepsilon_{0} R V^{2} \\
& {\left[\frac{1}{z}-\frac{1}{z+R\left(1-\sin \theta_{0}\right)}-\frac{1}{R} \ln \left(\frac{z+R\left(1-\sin \theta_{0}\right)}{z}\right)\right] } \\
F_{S}^{c}= & -\pi \varepsilon_{0} V^{2} \tan ^{2} \theta_{0} \\
& {\left[\ln \left(\frac{H}{z+R\left(1-\sin \theta_{0}\right)}\right)-1+\frac{R \cos ^{2} \theta_{0} / \sin \theta_{0}}{z+R\left(1-\sin \theta_{0}\right)}\right] }
\end{aligned}
$$

where the first expression represents the apex term, and the second one the shaft term, with $H$ being the shaft height. The $z$-derivative of such expressions is used to fit force gradient data, therefore the dependence on $H$ is eliminated.

The obtained best-fitting parameters are reported in table 2 . It is apparent how the probe radii results are sometimes different from the values visible in the SEM pictures, and in particular the shaft angles seem overestimated by this fitting. This is a demonstration of how the electrostatic measurement can give quite different results from those expected from the geometry derived by SEM imaging [26]. However, unrealistic values of angles suggest that there could be a systematic reason why the usual fitting can not appear to be accurate enough. We have concentrated on the fact that there might be a dependence on the finite oscillation amplitude $A_{0}$ used. Such an amplitude cannot be chosen to be too small during approach curve recording, in order to prevent damage of probes in the event that separation decreases too much, due for instance to unwanted thermal drifts. For our measurements, the amplitudes used were $A_{0}=16.5 \mathrm{~nm}$ for the probes ' $\chi$ ' and ' $\eta$ ', and $4 \mathrm{~nm}$ for the probe ' $\xi$ '.

To take into account the effect of large amplitudes, we have exploited an inversion formula after Sader and Jarvis [32], usually employed to carry out the force/distance dependence from AFM frequency shift data with an excellent precision, by taking into account the finite value of the oscillation amplitude. The correction provided by this formula is necessary when the oscillation amplitude spans a substantial part of the interaction potential, since in that case, the applied force changes considerably within the oscillation cycle. Here we apply such a method, for the first time to our knowledge, to electrostatic force gradient data. Such an inversion formula is expressed as:

$$
\begin{aligned}
F(z) & =\frac{2 k}{\omega_{0}} \int_{z}^{\infty} \\
& {\left[\left(1+\frac{A^{1 / 2}}{8 \sqrt{\pi(\zeta-z)}}\right) \Delta \omega(\zeta)-\frac{A^{3 / 2}}{\sqrt{2(\zeta-z)}} \frac{d \Delta \omega(\zeta)}{d \zeta}\right] d \zeta }
\end{aligned}
$$

where $\omega=2 \pi f$. This integration was performed numerically on our approach curve data $\Delta f(L)$.

Since the approach curves were acquired in a distance range of about $500 \mathrm{~nm}$, in order to perform the integration of equation (3) including the further data points, we have extrapolated the frequency shift data at large distance by an inverse power term of the form: 


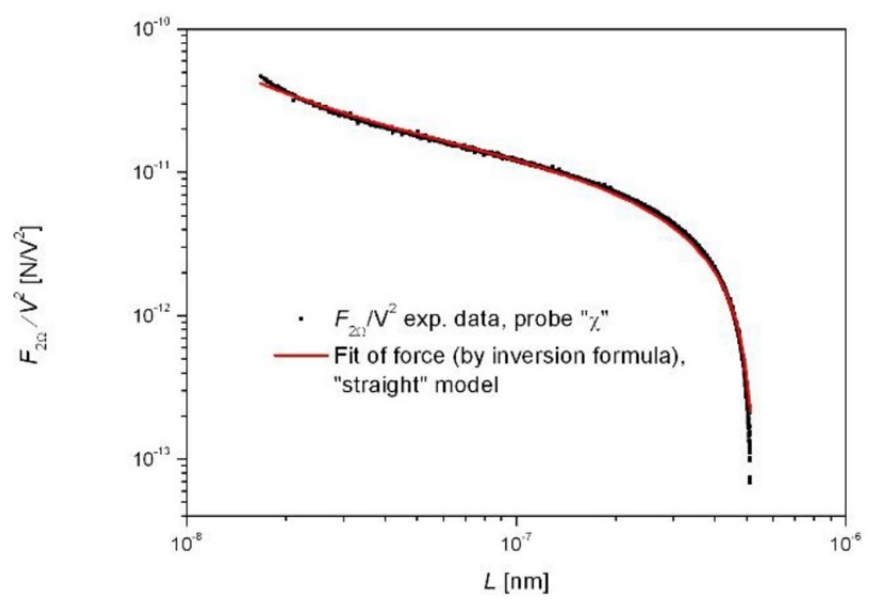

Figure 5. Approach curve for probe ' $\chi$ ', with best fit (red solid line) by the 'straight' model (equation (2)) for the force. Experimental data were converted from force gradient to force by resorting to the inversion formula of [31].

$$
\Delta \omega_{\infty}(z)=\frac{\alpha}{z+R\left(1-\sin \theta_{0}\right)}
$$

with the coefficient $\alpha$ adapted in order to join it with the acquired data at $z=500 \mathrm{~nm}$. This extrapolated function is also shown, as an illustration, for the case of the approach curve of figure 4.

The force curve obtained after the application of equation (3) is then best fitted by the 'straight' model for the force (equation (2)) [26]. Table 2 shows also the fitting results with the latter method, while figure 5 shows an example of inverted data, with the related best fit curve. It is apparent how fitting quality appears to be improved with respect to the case of the force gradient, and also how the values of shaft angles now look more realistic, while the values of the radii remain sufficiently close to the ones obtained by the previous method. We remark that the application of equation (3) provides a noticeable effect in such cases when the oscillation amplitude is comparable to the interaction range of electrostatic forces, that is, in our case, the tip radius $R$. Therefore, equation (3) is more necessary for tips ' $\chi$ ' and ' $\eta$ ', where the amplitude $A_{0}=16.5 \mathrm{~nm} \sim R$, while its effect is small for tip ' $\xi$ ', where $A_{0}=4 \mathrm{~nm} \ll R$.

\subsection{Lateral resolution assessment}

An example of an EFM image of our polymer multilayer sample is shown in figure 6 . The quality of the obtained crosssection is shown in the AFM topography map of figure 6(a), where the almost vertical line visible in the center is located in the proximity of the interface. The inclined lines are instead the scratches from the microtome knife. The corresponding electric contrast image is shown in figure 6(b), where the higher dielectric constant of PLA (2.3 at $80 \mathrm{~Hz}$ frequency and room temperature) compared to that of PS (2.0) translates into a higher electric signal (the brighter region). Dielectric constants differ by $15 \%$, while a contrast of $18 \%$ (lift mode) to $19.5 \%$ (CE-FM mode) was obtained in the electric images.
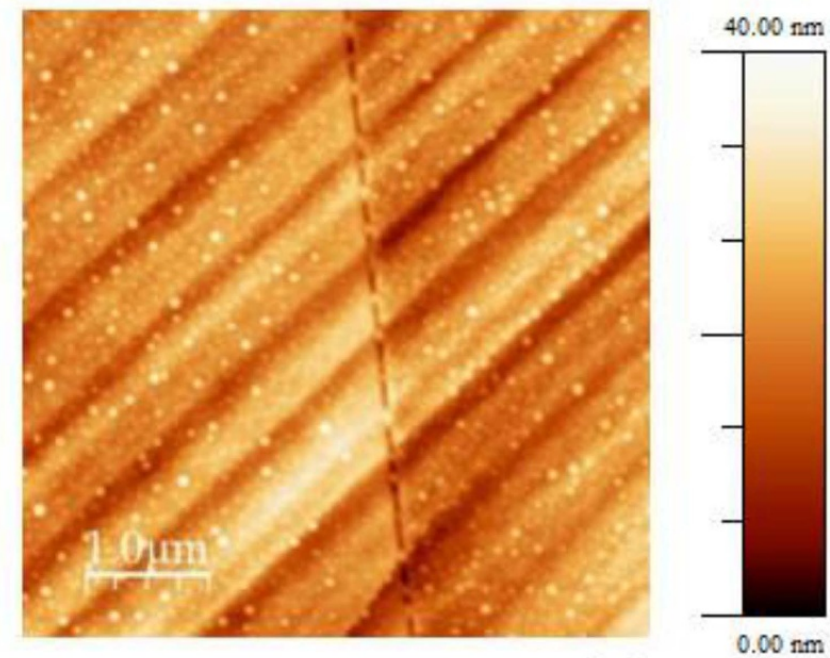

(a)
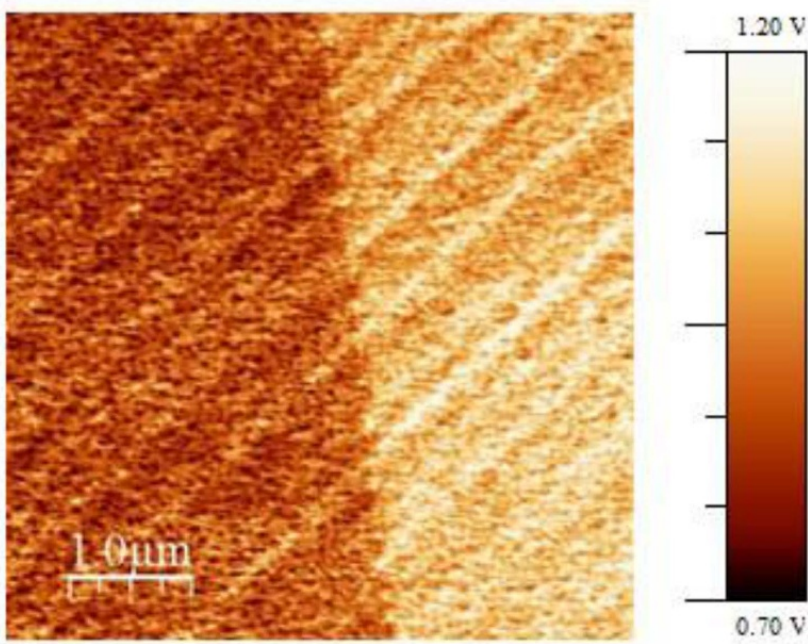

(b)

Figure 6. (a) Topography of the multilayered polymer sample. (b) Electric amplitude image (in arbitrary units). PLA (right side) exhibits higher polarizability than PS (left side). The diagonal lines indicate the scratches due to the microtome. The vertical line is located near the demarcation between the two polymers (PS and PLA). The scanning time for this image, acquired with an oscillation amplitude $A_{2}=4 \mathrm{~nm}$ in lift mode, was $4800 \mathrm{~s}$.

This difference is expected in EFM, since the capacitance depends on the dielectric constant in a non-linear way [26, 33].

An example of high resolution profile scanning across the interface is shown in figure 7(a) (topography) and figure 7(b) (electric). The total scan width was $1 \mu \mathrm{m}$, with 512 sampling points, for a limit point-to-point resolution of about $2 \mathrm{~nm}$. An example of the electric signal profile is also shown, along with the corresponding topographic trace (figure 7(c)), showing a roughness of about $2 \mathrm{~nm}_{\mathrm{RMS}}$. Sample flatness is important in order to reduce the effect of topography crosstalk on the EFM images [34].

Electric traces were fitted by a sigmoidal function:

$$
S(x)=a_{1}+\frac{a_{2}-a_{1}}{1+e^{-x / \delta_{s}}}
$$


(a)

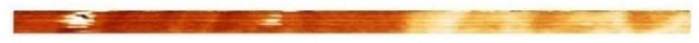

(b)

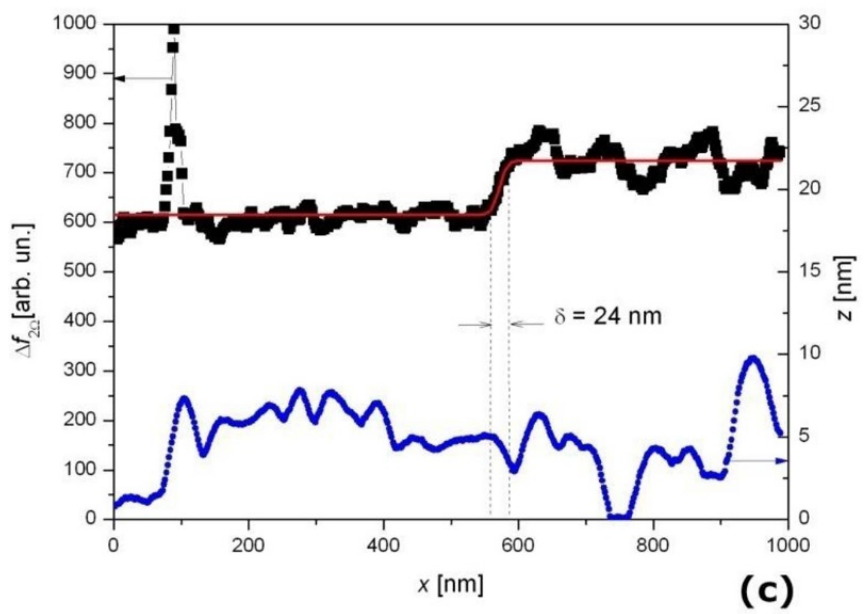

Figure 7. (a) Topography and (b) corresponding electric permittivity image of the test sample, obtained in lift mode by probe ' $\chi$ ', showing the sharp transition between the two polymers (images are shown just for illustration purposes. Image width is $1000 \mathrm{~nm}$, the scale bars are the same than in figure 6). The scanning time for these images, acquired with an oscillation amplitude $A_{2}=4 \mathrm{~nm}$ in lift mode, was $600 \mathrm{~s}$. (c) (top) Line profile taken from image (b), along with its fitting curve by the sigmoidal function of equation (5), showing dielectric contrast of $18 \%$ and lateral resolution of $24 \mathrm{~nm}$. (bottom) Line profile of topography, taken from image (a), showing poor correlation with the electric permittivity profile.

with the two asymptotic values $a_{1}$ and $a_{2}$ as well as the resolution $\delta_{\mathrm{s}}$ left as free fitting parameters (it can be shown that the $10 \%-90 \%$ resolution $\delta$ amounts in this case to $4.4 \delta_{\mathrm{s}}$ ). The fitting function plot is also reported in figure 7(c). The correspondence with the electric image is evident, while no relation with the topographic profile can be noticed.

The results of the profile fittings are shown in figure 8, for various distances, for the three tip models used. The resolution obtained in lift mode is found generally to be consistent, or even better, than the theoretical expectations of equation (1). The dashed lines in figure 8 report the prediction of equation (1), as calculated for the probe radius derived from the SEM images, while the dotted lines refer to the tip radii derived from our probe calibration procedures. For the first probe model (' $\chi$ '), oscillated with an amplitude $A_{2}=4 \mathrm{~nm}$ in lift mode, the experimental resolution (open symbols) is systematically better than predictions.

For the second probe model (' $\eta$ '), with $A_{2}=4 \mathrm{~nm}$, the agreement with the estimations is better. The third probe (' $\xi$ ') was oscillated with a smaller amplitude $\left(A_{2}=2 \mathrm{~nm}\right)$, and also provides good agreement. From these results, it could be concluded that theoretical estimations for constant-potential surfaces also describe with good accuracy the case of dielectric surfaces.

Numerical simulations of EFM resolution on thick dielectric samples [17] predict a linear trend with probe/sample distance, instead of a square root dependence such as
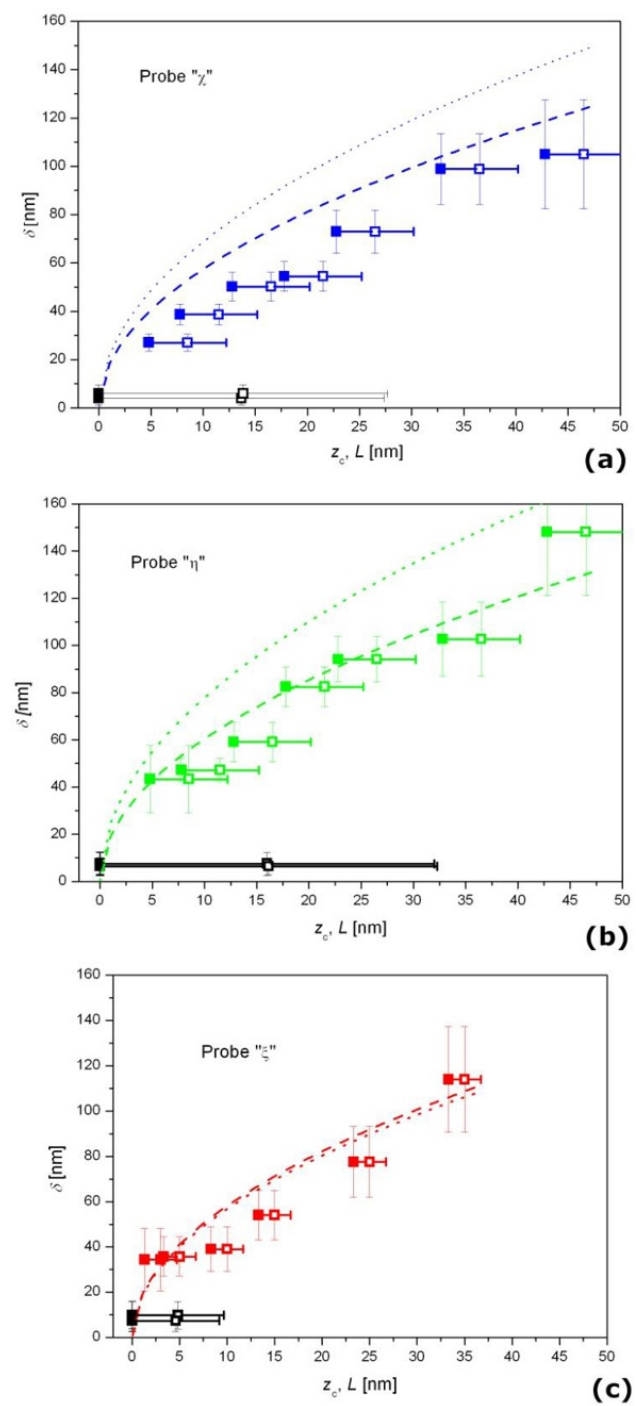

Figure 8. Resolution obtained in lift mode (colored hollow squares) and feedback-regulated mode (black hollow squares) as a function of separation $L$, for the three different probe types (see table 1): (a) probe ' $\chi$ ', (b) probe ' $\eta$ ', (c) probe ' $\xi$ '. The lines represent the $10 \%-90 \%$ resolution expected according to equation (1) and the tip radii derived from both SEM pictures (dashed line) and 'straight' force model (dotted line). Full symbols correspond to the same data plotted as a function of closest approach distance $z_{\mathrm{c}}$. Horizontal bars indicate the span of the used oscillation amplitude. Vertical bars indicate statistical errors on the fitting results, derived from averaging over five line profiles for each case.

equation (1). In particular, a finite value of resolution is predicted by such calculations, even at zero distance. By only considering the points obtained in lift mode, our results could also be consistent with such a trend, since they could also be fitted by a linear function. However, a further observation, which turned out to be the most important one in our investigation, concerns the fact that the resolution obtained in feedbackregulated mode looks far superior to that obtained in lift mode, at least for the cases considered here, and in contrast with the predictions above. As an example, line profiles similar to the ones of figure 7, but obtained in CE-FM mode, are shown in figure 9. The obtained resolution improvement compared to lift mode is visible. 
(a)

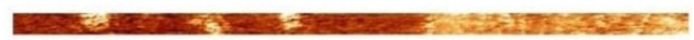

(b)

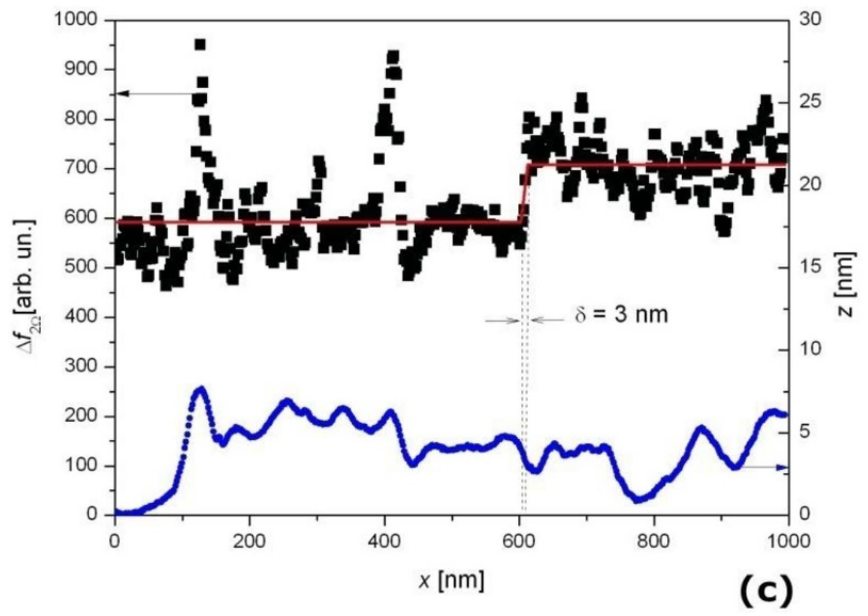

Figure 9. (a) Topography and (b) corresponding electric permittivity image of the test sample, obtained in CE-FM mode by probe ' $\chi$ ' (images are shown just for illustration purposes. Image width is $1000 \mathrm{~nm}$, the scale bars are the same than in figure 6). The scanning time for these images, acquired with an oscillation amplitude $A_{2}=16.5 \mathrm{~nm}$ in CE-FM feedback-regulated mode, was 300 s. (c) (top) Line profile taken from image (b), along with its fitting curve by the sigmoidal function of equation (5), showing dielectric contrast of $19.5 \%$ and lateral resolution of $3 \mathrm{~nm}$. (bottom) Line profile of topography taken from image (a), showing poor correlation with the electric permittivity profile.

By the results obtained, it can be speculated that the limit resolution might be ruled by the closest approach distance $\left(z_{\mathrm{c}}\right.$ in figure 2) rather than the average tip distance. To check this assumption, data in figure 8 were also plotted vs. $z_{\mathrm{c}}$ rather than vs. separation (full symbols). In this case, the correspondence of experimental data with the predictions (dashed and dotted lines) is improved. This is also the case for the lift mode.

It should be noticed that the value of $z_{c}$ in feedbackregulated mode is negligibly small compared to the oscillation amplitude, whereby $z_{\mathrm{c}}$ always reaches zero in figure 8 for data obtained in such a mode. However, even in this case, the obtained resolution in CE-FM mode does not look to be consistent with anticipations from the numerical simulations of [17]. The latter discrepancy could be related to the different definitions of resolution adopted, namely, the passage through a step boundary adopted here and in [15] vs. the width of a point-like object adopted in [17]. However, different causes for the observed resolution figures could also be possible. The roles of oscillation amplitude, closest approach distance, and feedback operation mode to determine EFM resolution on our dielectric test samples are currently under investigation.

\section{Conclusion}

We observed a dramatic spatial resolution improvement when imaging a step-like dielectric interface by EFM operated in feedback-regulated FM-AFM mode with constant oscillation amplitude, compared to the usual lift mode. This result suggests that closest approach distance $z_{\mathrm{c}}$ may rule the obtained resolution, rather than the average probe/surface distance (separation $L$ ), particularly in the feedback-regulated CE-FM operation mode. Such a conclusion is supported by comparison with theoretical estimations. Lateral resolutions from $3 \mathrm{~nm}$ (close to the resolution limit due to the sampling distance adopted in our experiment) to $10 \mathrm{~nm}$ were obtained under CEFM topography feedback, in spite of the oscillation amplitude used, of the order of 10-20 nm when operating in feedbackregulated mode. The achieved resolution range is of the order of typical correlation lengths in polymers $(2-3 \mathrm{~nm})[10,11]$, allowing more thorough investigations of dynamical heterogeneity effects in polymers in the real space [9].

\section{Acknowledgments}

We thank Randa Ishak (CISIM, University of Pisa) and CISUP (Centre for Instrumentation Sharing-University of Pisa) for SEM pictures of the tips, and Laurent Delbreilh (University of Rouen, France) for providing some preliminary test samples. M L acknowledges partial financial support from Office of Naval Research Global (grants VSP 18-7-002 and NICOP N62909-19-1-2121). R C acknowledges the support of the Office of Naval Research (N0001419WX00437) and CNR Short Term Mobility (STM2018 program) for the support of travel costs to Pisa. Image processing was performed by using WSxM freeware (http://wsxm.es).

\section{ORCID iDs}

M Labardi (D) https://orcid.org/0000-0003-4276-049X

R Casalini (D) https://orcid.org/0000-0002-5717-4103

\section{References}

[1] Cadena M J, Reifenberger R G and Raman A 2018 Nanotechnology 29405702

[2] Labardi M, Park J H, Nguyen H K, Prevosto D, Seong C Y, Mrzel A and Scalia G 2013 J Non-Cryst. Solids 379224

[3] Verdaguer A, Cardellach M, Segura J J, Sacha G M, Moser J, Zdrojek M, Bachtold A and Fraxedas J 2009 Appl. Phys. Lett. 94233105

[4] Nakagiri N, Yamamoto T, Sugimura H, Suzuki Y, Miyashita M and Watanabe S 1997 Nanotechnology 8 A32

[5] Crider P S, Majewski M R, Zhang J, Oukris H and Israeloff N E 2007 Appl. Phys. Lett. 91013102

[6] Riedel C, Arinero R, Tordjeman P, Ramonda M, Leveque G, Schwartz G A, de Oteya D G, Alegria A and Colmenero J 2009 J. Appl. Phys. 106024315

[7] Labardi M, Prevosto D, Nguyen H K, Capaccioli S, Lucchesi M and Rolla P A 2010 J. Vac. Sci. Technol. B 28 C4D11

[8] Labardi M, Lucchesi M, Prevosto D and Capaccioli S 2016 Appl. Phys. Lett. 108182906

[9] Oukris H and Israeloff N E 2010 Nat. Phys. 6135

[10] Casalini R, Zhu L, Baer E and Roland C M 2016 Polymer 88133 
[11] Fragiadakis D, Casalini R and Roland C M 2011 Phys. Rev. E 84042501

[12] Giessibl F J 2003 Rev. Mod. Phys. 75949

[13] Sadewasser S, Jelinek P, Fang C-K, Custance O, Yamada Y, Sugimoto Y, Abe M and Morita S 2009 Phys. Rev. Lett. $\mathbf{1 0 3} 266103$

[14] Zerweck U, Loppacher C, Otto T, Grafström S and Eng L M 2005 Phys. Rev. B 71125424

[15] Belaidi S, Lebon F, Girard P, Leveque G and Pagano S 1998 Appl. Phys. A 66 S239

[16] Derjaguin B V 1934 Kolloid Zeitschrift 69155

[17] Riedel C, Alegria A, Schwartz G A, Colmenero J and Saenz J J 2011 Nanotechnology 22285705

[18] Maragliano C, Heskes D, Stefancich M, Chiesa M and Souier T 2013 Nanotechnology 24225703

[19] Sadeghi A, Baratoff A and Goedecker S 2013 Phys. Rev. B 88035436

[20] Zhang G, Brannum D, Dong D, Tang L, Allahyarov E, Tang S, Kodweis K, Lee J-K and Zhu L 2016 Chem. Mater. 284646

[21] Colchero J, Gil A and Barò A M 2001 Phys. Rev. B 64245403

[22] Sacha G M, Cardellach M, Segura J J, Moser J, Bachtold A, Fraxedas J and Verdaguer A 2009 Nanotechnology 20285704
[23] Albrecht T R, Grütter P, Horne D and Rugar D 1991 J. Appl. Phys. 69668

[24] Gil A, Colchero J, Gomez-Herrero J and Barò A M 2003 Nanotechnology 14332

[25] Hudlet S, Saint Jean M, Guthman C and Berger J 1998 Eur. Phys. J. B 25

[26] Labardi M, Barsotti J, Prevosto D, Capaccioli S, Roland C M and Casalini R 2015 J. Appl. Phys. 118224104

[27] Bironeau A, Salez T, Miquelard-Garnier G and Sollogoub C 2017 Macromolecules 504064

[28] Riedel C, Sweeney R, Israeloff N E, Arinero R, Schwartz G A, Alegria A, Tordjeman P and Colmenero J 2010 Appl. Phys. Lett. 96213110

[29] Ueyama H, Sugawara Y and Morita S 1998 Appl. Phys. A $66 \mathrm{~S} 295$

[30] Protasenko V V, Labardi M and Gallagher A 2004 Phys. Rev. B 70245414

[31] Takeuchi O, Ohrai Y, Yoshida S and Shigekawa H 2007 Jpn. J. Appl. Phys. 465626

[32] Sader J E and Jarvis S P 2004 Appl. Phys. Lett. 841801

[33] Sacha G M, Sahagun E and Saenz J J 2007 J. Appl. Phys. 101024310

[34] Van Der Hofstadt M, Fabregas R, Biagi M C, Fumagalli L and Gomila G 2016 Nanotechnology 27405706 